\title{
Increasing oily fish consumption does not impact iodine intake or thyroid function in women of childbearing age
}

\author{
A.J. Yeates ${ }^{1}$, E. Kearney ${ }^{1}$, M. Conway ${ }^{1}$, T. Winter ${ }^{2}$, H. Below ${ }^{2}$, M.S. Mulhern ${ }^{1}$, \\ E.M. McSorley ${ }^{1}$ and J.J. Strain ${ }^{1}$ \\ ${ }^{1}$ Nutrition Innovation Centre for Food and Health (NICHE), Ulster University, Coleraine, UK and \\ ${ }^{2}$ Institute of Clinical Chemistry and Laboratory Medicine, University Medicine Greifswald, Germany
}

Mild to moderate iodine deficiency is a public health challenge in many countries. Insufficient intakes of iodine, which is essential for thyroid hormone production, in pregnancy and indeed, prior to conception, may have negative consequences for early brain development $^{(1)}$. Lean seafood intake has been shown to increase urinary iodine concentrations (UIC), with no available data for oily fish ${ }^{(3)}$. The aim of this study was to investigate the effects of increasing oily fish consumption on UIC compared to no fish consumption among women of childbearing age.

Data were analysed from a randomised controlled trial where women of childbearing age were randomly assigned to one of three groups, to consume no fish $(n=18), 1$ portion $(n=14)$ or 2 portions $(n=17)$ of tuna and sardines per week for an 8 -week period ${ }^{(4)}$. Retrospective power calculations indicated sufficient sample size to investigate the current study aim. At weeks 0 and 8 participants, who were habitually low consumers of fish at baseline $(<2$ portions/wk), provided a fasting blood and spot urine sample. Samples were shipped to the University of Greifswald for measurement of UIC, a biomarker of iodine intake, using the Sandell-Kolthoff reaction. Markers of thyroid function (serum free concentrations of T3, T4 and thyroid stimulating hormone (TSH) and dried blood spot thyroglobulin) were also measured by chemiluminescent immunoassay. To test the effect of intervention on UIC, analysis of covariance was used adjusting for age and baseline UIC. Chi-square test was used to compare UIC between groups when categorised as insufficient/ sufficient $(</ \geq 100 \mu \mathrm{g} / \mathrm{L})$ according to World Health Organisation (WHO) criteria $^{(6)}$.

Forty-six participants completed the intervention with a mean \pm SD age of $25.4 \pm 6.5$ years. Median (IQR) UIC of the cohort at baseline was $128.64(71.41-267.44) \mu \mathrm{g} / \mathrm{L}$, indicating sufficient iodine intake according to WHO criteria. There was no difference in UIC between groups at baseline. Post-intervention there was no significant difference in UIC between groups who consumed one (median (IQR) $130.59(30.39-229.31) \mu \mathrm{g} / \mathrm{L})$ or two portions of fish $(133.69(73.64-173.86) \mu \mathrm{g} / \mathrm{L})$ per week compared to the control $(99.05(84.98-185.19) \mu \mathrm{g} / \mathrm{L})(\mathrm{F}(2)=0.007, \mathrm{P}=0.99)$. Neither were there any differences in markers of thyroid function between groups $(\mathrm{P}>0.05)$. Following the intervention, the proportion of those with sufficient UIC $(>100 \mu \mathrm{g} / \mathrm{L})$ was greater within the two portions of fish group, but this did not reach significance $(\mathrm{x} 2=5.28, \mathrm{P}=0.07)$.

Increasing oily fish consumption to 1 or 2 portions per week did not significantly increase UIC among women of childbearing age compared to no fish consumption. This cohort had sufficient iodine intakes and normal thyroid function. Other studies are needed to investigate the contribution of dietary sources to iodine intakes among women of childbearing age who are at most risk of iodine deficiency.

\section{References}

1. Bath SC, Steer CD, Golding J, et al. (2013) The Lancet 382(9889), 331-337

2. Øyen J, Aadland EK, Liaset B, et al. (2021) Eur J Nutr 60, 1679-1689

3. Conway M, McSorley EM, Mulhern MS et al. (2021) Eur J Nutr 60, 1415-1427

4. World Health Organization (2007) Pub Health Nutr 10 (12A), 1606-1611 